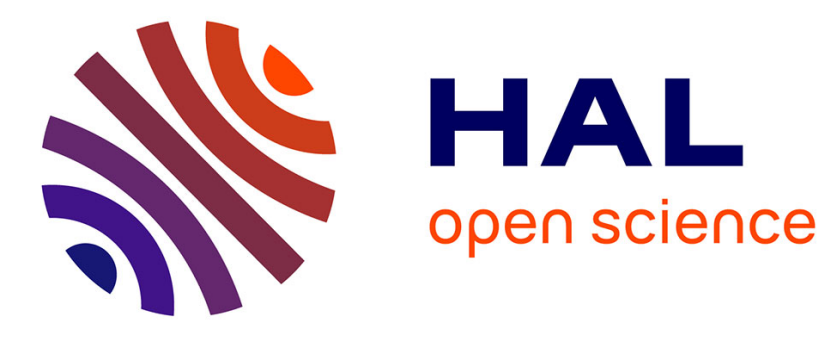

\title{
Water-Dispersed High-Quality Graphene: A Green Solution for Efficient Energy Storage Applications
}

Zhaoyang Liu, Heng Zhang, Matilde Eredia, Haixin Qiu, Walid Baaziz, Ioan-Ovidiu Ersen, Artur Ciesielski, Mischa Bonn, Hai I. Wang, Paolo Samori

\section{- To cite this version:}

Zhaoyang Liu, Heng Zhang, Matilde Eredia, Haixin Qiu, Walid Baaziz, et al.. Water-Dispersed HighQuality Graphene: A Green Solution for Efficient Energy Storage Applications. ACS Nano, 2019, 13 (8), pp.9431-9441. 10.1021/acsnano.9b04232 . hal-03022788

\section{HAL Id: hal-03022788 https://hal.science/hal-03022788}

Submitted on 24 Nov 2020

HAL is a multi-disciplinary open access archive for the deposit and dissemination of scientific research documents, whether they are published or not. The documents may come from teaching and research institutions in France or abroad, or from public or private research centers.
L'archive ouverte pluridisciplinaire HAL, est destinée au dépôt et à la diffusion de documents scientifiques de niveau recherche, publiés ou non, émanant des établissements d'enseignement et de recherche français ou étrangers, des laboratoires publics ou privés. 


\title{
Water-Dispersed High-Quality Graphene: A Green
}

\section{Solution for Efficient Energy Storage Applications}

\author{
Zhaoyang Liu, ${ }^{1}$ Heng Zhang, ${ }^{2}$ Matilde Eredia, ${ }^{1}$ Haixin Qiu,${ }^{1}$ Walid Baaziz, ${ }^{3}$ Ovidiu Ersen, ${ }^{3}$ \\ Artur Ciesielski, ${ }^{I *}$ Mischa Bonn, ${ }^{2}$ Hai I. Wang, ${ }^{2}$ Paolo Samori ${ }^{1 *}$
}

${ }^{1}$ Université de Strasbourg and CNRS, ISIS, 8 allée Gaspard Monge, 67000 Strasbourg, France.

${ }^{2}$ Max Planck Institute for Polymer Research, Ackermannweg 10, 55128 Mainz, Germany

${ }^{3}$ Institut de Physique et Chimie des Matériaux de Strasbourg (IPCMS), UMR 7504 Université de Strasbourg and CNRS, 23 rue du Loess, 67034 Strasbourg, France

*corresponding authors: ciesielski@unistra.fr; samori@unistra.fr

KEYWORDS: solution-processable, water-dispersed graphene, high-quality, electrochemical exfoliation, micro-supercapacitors

\section{ABSTRACT}

Graphene has been the subject of widespread research during the last decade because of its outstanding physical properties which make it an ideal nanoscale material to investigate fundamental properties. Such unique characteristics promote graphene as a functional material for the emergence of new disruptive technologies. Yet, to impact our daily-life products and devices, high-quality graphene needs to be produced in large quantities, using an environmentally-friendly protocol. In this context, the production of graphene which preserves its outstanding electronic properties using a green chemistry approach remains a key challenge. 
Herein, we demonstrate an efficient electrochemical approach to obtain water-dispersed highquality graphene nanosheets. High-frequency (terahertz) conductivity measurements of the graphene nanosheets reveal high charge carrier mobility up to $1000 \mathrm{~cm}^{-2} \mathrm{~V}^{-1} \mathrm{~s}^{-1}$. The fine water dispersibility enables versatile functionalization of graphene, as demonstrated by the pseudocapacitive polydopamine coating of graphene nanosheets. Remarkably, the polydopamine functionalization causes a modest, i.e. $20 \%$, reduction of charge carrier mobility. Thin film electrodes based on such hybrid materials for micro-supercapacitors exhibit excellent electrochemical performance, namely high volumetric capacitance of $340 \mathrm{~F} \mathrm{~cm}^{-3}$ and power density of $1000 \mathrm{~W} \mathrm{~cm}{ }^{-3}$, thus outperforming most of the reported graphene-based microsupercapacitors. These results highlight the potential for water-dispersed, high-quality graphene nanosheets as a novel materials platform for energy storage applications.

\section{Introduction}

To address the ever-increasing energy demands in diverse technologies to improve the quality of our lives, the development of reliable and Eco-friendly approaches for energy storage systems (ESSs) has become one of the major challenges for the society. ${ }^{1}$ In particular, the next generation of wearable electronics will require the use of light-weight, flexible, and highly-conductive materials. Graphene, which is the ancestor of the growing family of two-dimensional atomic crystals, is being regarded as a rising star material for ESSs, because it combines very high mechanical robustness, excellent electrical and thermal conductivity, large specific surface area, and high intrinsic areal capacitance. ${ }^{2}$ Among current ESSs, micro-supercapacitors (MSCs) with planar architectures have recently emerged as potential energy source for future generation of on-chip miniaturized electronics in a flexible or even wearable form, since they are capable of 
sustaining high power, fast charging/discharging and long cycling lives, without the safety concerns as for batteries. ${ }^{3}$

In recent years, different strategies have been pursued to achieve interdigitated electrode arrays utilizing graphene or graphene-based hybrid materials. These attempts have been based primarily on employing graphene oxide (GO) as a precursor for fabricating graphene-based MSCs. This trend can be explained by the fact that GO, rather than graphene itself, can be produced via lowcost and scalable wet-chemical approaches, and perhaps even more importantly, once produced, GO can be subsequently functionalized to combine pseudocapacitive materials such as metal oxide nanoparticles or conducting polymers. ${ }^{4-8}$ Advanced techniques to subsequently convert GO into graphene, include laser scribing, which directly converts GO into graphene, ${ }^{9-11}$ selective electrophoretic deposition of GO followed by chemical reduction, ${ }^{12}$ and methane plasma treatments which can partially repair the defects of GO ${ }^{13}$.

However, reduced graphene oxide (RGO) constitutes moderate-quality graphene, given the presence of abundant defects (at both edges and surfaces of RGO nanosheets). As a result, RGO does not exhibit graphene's exceptional electronic properties, which cannot indeed be fully restored even after (electro)chemical/thermal reduction. Moreover, the re-stacking of RGO in water is another fatal drawback, which can cause a severe decrease of the electrochemically accessible surface area, which limits the overall performance of graphene-based MSCs. Therefore, the production of solution-processable high-quality graphene nanosheets via a scalable approach is highly desirable. Recent studies in electrochemical exfoliation of graphite highlight the potential of such a method as an efficient wet chemical approach to produce highquality graphene in large quantities. ${ }^{14}$ Due to the fast exfoliation process, only minor oxidation occurs at the graphite electrode. Notably, the absence of functional groups on the obtained 
electrochemically exfoliated graphene (EG) surface makes them only dispersible in high boiling point and often highly toxic organic solvents such as $\mathrm{N}$-methyl-2-pyrrolidone (NMP) or $\mathrm{N}, \mathrm{N}$ dimethylformamide (DMF). This issue limits the subsequent processing and functionalization of $\mathrm{EG}$, and the use of toxic organic solvents may raise both environmental and health concerns. Thus, the fabrication of high-quality graphene via environmentally friendly approaches is highly desirable.

Herein, we report the efficient production of water-dispersed high-quality graphene nanosheets by electrochemical exfoliation. The multiscale characterization of the physicochemical properties of the obtained graphene nanosheets revealed a low oxygen content of 12 atom $\%$ as determined by X-ray Photoelectron Spectroscopy (XPS), a low ratio between 2D band and G band as evidenced by Raman spectroscopy, a high electrical conductivity of $340 \mathrm{~S} \mathrm{~cm}^{-1}$ as measured by a four-probe system, and a high charge carrier mobility up to $1000 \mathrm{~cm}^{-2} \mathrm{~V}^{-1} \mathrm{~s}^{-1}$ as quantified by Terahertz measurements. Such characteristics provide unambiguous evidence for the preserved high-quality nature of obtained EG nanosheets. Morphology studies by atomic force microscopy (AFM) and high-resolution transmission electron microscopy (HR-TEM) indicate that the obtained EG are dominated by bi-layer flakes. In order to boost the overall MSC device performance, the as-produced EG nanosheets were further functionalized by pseudocapacitive polydopamine (PDA). After PDA functionalization, the intrinsic electrical transport properties of EG studied by THz spectroscopy are found to be well preserved (with only a minor decrease of conductivity of $\sim 20 \%$ ). PDA-EG based electrodes for MSCs exhibited high performance, with a volumetric capacitance of $340 \mathrm{~F} \mathrm{~cm}^{-3}$ and a high-power density of $1000 \mathrm{~W} \mathrm{~cm}^{-3}$, which outperformed most of the reported values of graphene-based MSCs. The MSC device also allows 
ultrahigh speed operations up to $1000 \mathrm{~V} \mathrm{~s}^{-1}$, being three orders of magnitude higher than conventional sandwich-structured electric double-layer capacitors.

\section{Results and discussion}

Figure 1 illustrates the general procedure employed towards water-dispersed electrochemically exfoliated graphene (EG) nanosheets. A cryogenic pretreatment was first applied to graphite foil, by soaking it alternatively in liquid nitrogen and absolute ethanol (Figure 1a), during which, both the violent nitrogen evolution and the ethanol solidification process weakens the interlayer van der Waals interactions between the graphene layers and result in the volume expansion by nearly 10 times. Meanwhile, a large number of small cracks are formed at the graphite intralayer grain boundaries, due to the drastic volume change. The volume expansion, as well as the generated cracks, provides certain pathways for capillary effects, which facilitate the subsequent intercalation of anions within the graphite layers during electrochemical exfoliation. ${ }^{15-17}$ The electrochemical exfoliation of graphite was conducted by a custom-made two electrode system, where graphite foil was acting as anode and platinum foil was acting as a cathode (Figure 1b), driven by direct current voltage $(10 \mathrm{~V})$. An aqueous solution of $0.1 \mathrm{M} \mathrm{NaOH}$ was employed as an electrolyte, which is capable of exfoliating graphite into graphene after a long period (2h), as witnessed by the gradual darkening of the solution. After washing and mild sonication, the obtained graphene can be finely dispersed in water. Typically, a slow electrochemical exfoliation is attributed to the insufficient anion intercalation within the graphite layers, and in general, the obtained material exhibits increased levels of oxygen. To improve the exfoliation process and obtain graphene nanosheets with lower oxygen content, we propose a mixed electrolyte approach, as shown in Figure 1b. Although the overall mechanism for electrochemical exfoliation of graphite is not fully unraveled, sulfate anions are commonly suggested to be 
efficient for graphite intercalation under certain bias. ${ }^{18-19}$ Here, we propose a new approach, in which small amount of sulfate anions is used to intercalate graphite foil and thus makes easier access for hydroxyl anions, which subsequently exfoliate the material and ultimately graphene nanosheets are obtained. An aqueous solution of $0.1 \mathrm{M} \mathrm{Na}_{2} \mathrm{SO}_{4}$ was thus mixed with $\mathrm{NaOH}$ and the ratio was carefully controlled to optimize the quality of exfoliated material. The optimal ratio between $\mathrm{NaOH}$ and $\mathrm{Na}_{2} \mathrm{SO}_{4}$ was determined to be 10:1, which allows the complete exfoliation of graphite foil (thickness around $1.3 \mathrm{~mm}$ after pretreatment) within 30 minutes. When the $\mathrm{Na}_{2} \mathrm{SO}_{4}$ content is increased to, for example, 5:1, the exfoliation process is accelerated and finishes within 12 minutes. However, the obtained EG cannot be well dispersed in water due to insufficient oxygen content. On the other hand, when the amount of $\mathrm{Na}_{2} \mathrm{SO}_{4}$ is reduced to, for example, 25:1, the exfoliation is slowed down and takes 1 hour. Moreover, when $\mathrm{NaOH}$ alone is employed as an electrolyte, the exfoliation duration was found to exceed 3 hours.

Figure 1c shows the photograph of a stable water dispersion of the as produced EG ink. In order to confirm the colloidal nature of the dispersion, we performed the Tyndall effect and salt effect, as well as the Zeta potential measurements, which are typically conducted for colloidal dispersions. As shown in Figure 1d, a diluted EG dispersion exhibits significant Tyndall effect, where a laser beam leaves a clear distinguishable light track due to scattering. Adding salt $(\mathrm{NaCl}$ solution) to the EG water dispersion leads to fast aggregations of EG flakes (Figure 1e). The obtained Zeta potential values based on these three conditions are all lower than $-35 \mathrm{mV}$ (Fig. S1 in the Supporting Information), suggesting sufficient electrostatic repulsion which explains the high stability of the EG aqueous dispersion. ${ }^{20}$

Such strong electrostatic repulsions can be attributed to the ionization of the oxygen-containing groups on EG surface, which is studied by X-ray photoelectron spectroscopy (XPS) as shown in 
Figure 2a. Similarly to the case of graphene oxide (GO) with well-known bonds composition, the EG surface exhibits minor oxidized species, i.e., carboxylic and hydroxyl groups, introduced during the electrochemical exfoliation process. However, the total oxygen content of EG is only $12 \%$, being comparatively lower than that of GO and thermally reduced graphene oxide (TRGO), which exhibit an oxygen content of $31 \%$ and 14\%, respectively (Fig. S2 and S3). Here the comparison of oxygen content is confined to water-dispersible derivatives of graphene, rather than EG obtained via fast electrochemical exfoliation exhibiting lower oxygen content, which can only be dispersed in high-boiling point organic solvents. This comparison suggests that the obtained water-dispersed EG should exhibit largely preserved electronic properties, which, based on the oxygen content, are expected to outperform TRGO. Moreover, the thermal reduction treatment of our EG $\left(500^{\circ} \mathrm{C}\right.$ under vacuum $)$ removes the hydroxyl groups significantly and results in very low oxygen content of only $4.8 \%$, being even lower than the theoretical limit for RGO after $1500 \mathrm{~K}$ annealing. ${ }^{21-22}$ Such direct comparison suggests the enormous potential of our water-dispersible EG in replacing RGO as solution-processed high-quality graphene for future perspectives.

Raman spectroscopy is subsequently employed to identify the quality of EG further, as shown in Figure 2b. A dispersion containing EG nanosheets is drop-cast on $\mathrm{SiO}_{2} / \mathrm{Si}$ substrate. The narrow $\mathrm{G}$ band at $1584 \mathrm{~cm}^{-1}$ corresponds to the first-order scattering of the $\mathrm{E}_{2 \mathrm{~g}}$ vibrations of the graphene lattice. An intense and symmetric 2D band at around $2710 \mathrm{~cm}^{-1}$ suggests the nature of few-layer graphene (2-4 layers), which could be due to the reaggregation during sample preparation. Surprisingly, the intensity ratio between the $2 \mathrm{D}$ and $\mathrm{G}$ bands $\left(\mathrm{I}_{2 \mathrm{D}} / \mathrm{I}_{\mathrm{G}}\right)$ amounts to 0.7 , being comparable to that of microwave reduced GO, and is larger than chemically/thermally reduced GO, liquid phase exfoliated graphene, and HOPG, indicating the high quality of our EG. ${ }^{21}$ 
Meanwhile, a pronounced D band at $1354 \mathrm{~cm}^{-1}$ is observed, which can be ascribed to the presence of defects, such as edges and unavoidable oxidation during the deliberately prolonged electrochemical exfoliation process. The intensity ratio between $\mathrm{D}$ band and $\mathrm{G}$ band $\left(\mathrm{I}_{\mathrm{D}} / \mathrm{I}_{\mathrm{G}}\right)$ amounts to 1.3 , being comparable to the typically reported electrochemical exfoliation with sulfuric acid as electrolyte ${ }^{23}$, and even smaller than some salt based electrolyte case ${ }^{24-25}$ and $\mathrm{TRGO}^{26}$.

The results of the statistical analysis of EG thickness distribution carried out by AFM are portrayed in Figure 2c and d. It reveals a predominance of sheets exhibiting a thickness of 1.60 $\mathrm{nm}$, concluded to be bilayer graphene in accordance with the thickness of freshly cleaved singlelayer graphene measured on $\mathrm{SiO}_{2}$ substrates $(0.6-0.8 \mathrm{~nm}) .{ }^{27}$ A cross-sectional analysis of $60 \mathrm{EG}$ nanosheets suggests that most of the obtained EG flakes are thinner than $3 \mathrm{~nm}$, among which about $60 \%$ of the sampled graphene sheets has a thickness between 1 and $2 \mathrm{~nm}$. Thus, our obtained EG nanosheets are mainly composed of bilayer graphene. Scanning electron microscopy (SEM) reveals the prevalent lateral-size of EG nanosheets is 2-4 $\mu \mathrm{m}$ (Figure $2 \mathrm{~g}$ ). Transmission electron microscopy (TEM) revealed certain overlapping between the EG nanosheets (Figure 2h), which originates from the drying process during sample preparation. The high-resolution TEM further confirmed the bilayer nature of EG (Figure 2e). The selected-area electron diffraction (SAED) pattern of EG exhibits a typical 6-fold symmetry (Figure 2f), characterized by stronger diffraction from the (1-210) diffraction spot than (0-110), demonstrating the high crystallinity nature of bilayer graphene. The electrical conductivity measurement shows a significant conductivity of $340 \mathrm{~S} \mathrm{~cm}^{-1}$ based on EG films (Fig. S4), suggesting the preserved electronic properties. 
The fine dispersibility and preserved properties make our EG a promising material for energy storage-related devices, especially to fabricate MSCs based on graphene inks. Highly concentrated EG dispersions (10 $\mathrm{mg} \mathrm{ml}^{-1}$ in isopropanol) can be employed as printable inks to achieve large area in-plane MSCs on paper substrates, by spray-coating through a shadow mask (Fig. S5a). The printed patterns of EG shows a significant low resistance of $7 \Omega^{-1}(\sim 3 \mu \mathrm{m}$ thick film), which is superior to that of state-of-the-art carbon nanotube-based conductive papers (10 $\Omega{ }^{-1}$ based on several $\mu \mathrm{m}$ thick film $)^{28}$. The low resistance of EG patterns makes the use of metal (typically gold) current collectors unnecessary, which simultaneously reduces the cost of the device and demonstrates the possibility of future scalable production of flexible/wearable MSCs arrays based on EG. However, being only capable of electrical-double-layer capacitance without exhibiting pseudocapacitance behavior, EG's electrochemical performance in MSCs is limited (Fig. S5 b-d). A maximum volumetric capacitance of $53 \mathrm{~F} \mathrm{~cm}^{-3}$ is delivered at a low scan rate of $1 \mathrm{mV} \mathrm{s}^{-1}$, decreasing significantly when the scan rate is increased to $100 \mathrm{mV} \mathrm{s}^{-1}$, which is far from satisfactory.

Quinones have been already studied as stable and highly capacitive electrode materials for aqueous rechargeable batteries, due to the presence of fast and reversible redox reactions..$^{20-21}$ Inspired by such promising results, we decided to further functionalize our EG nanosheets with polydopamine coatings to enhance the performance of EG-based MSCs. Dopamine is added into the $\mathrm{EG}$ dispersion at $\mathrm{pH} 8.5$, and the polymerization is completed within 24 hours. After the polydopamine coating, PDA-EG shows a significant increase in the nitrogen and oxygen content, as expected from the presence of PDA (Fig. S6). The AFM reveals an average thickness of $\sim 6$ nm of PDA-EG, demonstrating the uniform distribution of PDA coating, which can also be observed by HR-TEM of the PDA-EG single flake (Fig. S7). The SEM study shows the average 
lateral size of PDA-EG is 2-4 $\mu \mathrm{m}$, inheriting from the EG flake size distribution. The SAED pattern of PDA-EG displays the strong diffraction being nearly identical to the neat EG, thereby suggesting that the PDA coating does not influence the high crystallinity of EG and its associated electronic properties.

To investigate the charge transport properties and the influence of PDA coating on the electronic properties of the electrochemically exfoliated graphene, optical pump-Terahertz $(\mathrm{THz})$ probe (OPTP) spectroscopy is employed. As a purely optical approach, THz spectroscopy can provide quantitative insight into the intrinsic charge transport properties (e.g., charge mobility, carrier density, etc.) in the materials of interests in a contact-free fashion, ${ }^{29-33}$ which is particularly powerful for accessing the electrical properties of low-dimensional materials where electrical contacts are often challenging to deposit for conventional electrical studies. In a typical OPTP measurement, the EGs deposited on the fused silica (using the Langmuir-Blodgett method) are photoexcited by a short femtosecond ( $\sim 50 \mathrm{fs}$ ) laser pulse with a photon energy of $\sim 3.1 \mathrm{eV}$ (400 $\mathrm{nm}$ ) to generate charge carriers. Subsequently, a single-cycle, freely propagating pulse at $\mathrm{THz}$ frequencies (of $\sim 1$ ps duration) interacts with the charge carriers and reports back on their photoconductivity in the $\mathrm{THz}$ frequency range (as shown in Figure 3(a)). The strength of $\mathrm{THz}$ spectroscopy is that it records the time-dependent field $E(t)$ of the THz pulse, rather than only its intensity, at different times after the photoexcitation pulse. It can be shown that the pumpinduced change of the $\mathrm{THz}$ field $\Delta E(t)$ normalized to the transmitted $\mathrm{THz}$ field before excitation $E$ is directly proportional to the real part of the THz photoconductivity $\sigma: \sigma \sim-\Delta E / E$. THz spectroscopy has been intensively used to characterize charge transport in graphene. ${ }^{29,31,34}$ Figure $3 \mathrm{~b}$ shows the short-lived (picosecond) photoconductivity dynamics of EG and PDA-EG films. The photoexcitation causes injection of charge carriers into the conduction band in the multilayer 
EG sheets, which recombine quickly. The measured conductivity is determined by the injected charge carrier density $N_{\text {pump }}$, charge mobility $\mu$, and elemental charge $e: \sigma=N_{\text {pump }} * e^{*} \mu$. To allow for a direct comparison of charge transport properties between EG and PDA-EG, THz conductivities of both samples have been normalized to the absorbed photon density $N_{A b s}$, which, assuming that the quantum efficiency is identical for the two samples, is proportional to $N_{\text {pump. }}$. In this manner, the relative change in conductivities directly reflects the change in charge mobility in EG by PDA treatment. As clearly shown in Figure 3b, the electronic properties of the EG samples after PDA coating are well-preserved with at most a $\sim 20 \%$ decrease in $\mathrm{THz}$ conductivity for PDA-EG compared to that of EG.

To quantify the electrical transport of photogenerated carrier in EG and PDA-EG, we have determined the complex THz photoconductivity spectra of the samples $\sim 600$ fs after excitation (details see SI). As shown in Figure 3 (c-d), the measured conductivity is characterized by positive real photoconductivity and negative imaginary conductivity, both increasing in absolute magnitude with increasing frequency. This type of response is characteristic of charge carriers in low-dimensional systems and can be described by the Drude-Smith (DS) model (lines in Figure 3 (c-d)). The DS model is a phenomenological model which has been successfully applied to describe transport in low-dimensional, confined systems. ${ }^{35-36}$ As shown in Figure $3 \mathrm{c}$ and $3 \mathrm{~d}$, the experimental data can be adequately fitted by Drude-Smith model, which reveals the scattering time $\tau_{e}$ of $\sim 18$ fs and $\sim 14$ fs for EG and PDA-EG, respectively. The plasma frequencies $\omega_{p}$ normalized to the absorbed photon density are found to be nearly identical (with $\omega_{p}^{E G}=187.1$ THz for absorbed phtodensity of $7.2 * 10^{12} \mathrm{~cm}^{-2}$ and $\omega_{p}^{P D A-E G}=230.7 \mathrm{THz}$ for absorbed photon density of $8.9 * 10^{12} \mathrm{~cm}^{-2}$ ), which is in agreement with our previous assumption of the same quantum yields of free carrier generation in both samples by photo-excitation. Furthermore, 
employing the effective electron mass $m^{*}$ of $\sim 0.03 \mathrm{~m}_{\mathrm{e}}$ reported in the literature for bilayer graphene, the mobility $\left(\mu=\frac{e * \tau_{e}}{m *}\right)$ in EG can reach up to 1100 and $860 \mathrm{~cm}^{2} \mathrm{~V}^{-1} \mathrm{~s}^{-1}$ for $\mathrm{EG}$ and PDA-EG, respectively. These high mobilities in graphene produced by our electrochemical exfoliation method, illustrate the high quality of the EG flakes. Additionally, the inferred mobility in PDA-EG is concluded to be $22 \%$ lower that of EG sample, in a perfect agreement with the dynamic terahertz scans (Figure 3(a)) that revealed a $\sim 20 \%$ reduction of conductivity in EG after PDA coating treatment.

Although in general, the functionalization of graphene and other two-dimensional materials with organic or inorganic molecules typically leads to the slight decrease of the electronic properties (e.g., decrease of the conductivity) of 2D nanosheets due to the formation of in-plane voids and cavities, such behavior is not considered as a drawback. In fact, many reports have suggested that the presence of structural and chemical defects improves the overall electrochemical performance of 2D nanosheets. ${ }^{37}$ In order to highlight the electrochemical properties of PDA-EG as a supercapacitor electrode material, we fabricated on-chip in-plane MSCs based on thin PDAEG films on paper substrates with channels much shorter than those of spray-coated EG (Fig. S5a), the scheme being shown in Figure 4a. Briefly, the as-synthesized PDA-EG was washed repeatedly to remove the tris buffer and unreacted dopamine molecules. Upon subsequent gentle sonication, the PDA-EG flakes were finely dispersed in isopropanol (IPA). Uniform PDA-EG films can be readily obtained on a filter membrane by vacuum filtration of the PDA-EG dispersions. Finally, the PDA-EG films were pressed against target substrates (precleaned $\mathrm{SiO}_{2} / \mathrm{Si}$ ) and kept for 2 hours with the applied pressure. Such "dry transfer" technique provides a uniform and clean approach to obtain PDA-EG films with controlled thickness. The MSC devices were fabricated using well-established lithography techniques. The interdigitated 
microelectrode patterns were deposited onto the PDA-EG films through a home-made shadow mask, with gold as current collectors (see Fig. S8 for the detailed device geometry). Oxygen plasma was applied to etch away the unprotected part of the film to achieve the desired patterns. A polymer gel electrolyte based on $\mathrm{H}_{2} \mathrm{SO}_{4} /$ polyvinyl alcohol $\left(\mathrm{H}_{2} \mathrm{SO}_{4} / \mathrm{PVA}\right)$ was subsequently drop-cast onto the interdigitated microelectrodes and solidified overnight. The use of gel electrolyte avoids leakage and additional packaging issues of liquid electrolytes. Thus, all solidstate MSCs based on PDA-EG with an in-plane device geometry were realized.

To evaluate the electrochemical performance of PDA-EG based MSCs, cyclic voltammetry (CV) was performed at different scan rates (Figure 4b-g). Noteworthy, such microdevices demonstrate ultrafast charging/discharging capability and can be operated at ultrahigh speed up to $1000 \mathrm{~V} \mathrm{~s}^{-1}$, being three orders of magnitude greater than conventional sandwiched-structured supercapacitors.$^{38}$ Compared with the aforementioned MSCs based on pristine EG, PDA-EG based MSCs (PDA-EG-MSC) show significantly enhanced electrochemical performance. The $\mathrm{CV}$ curves are nearly rectangular shaped between the voltage potential of 0 and $1 \mathrm{~V}$, even at a high scan rate of $100 \mathrm{~V} \mathrm{~s}^{-1}$. This indicates the fine combination of both large electrical-doublelayer capacitance from EG and pronounced pseudocapacitance from PDA. Real applicable devices thus prove the undisrupted crystal structures and preserved electronic properties of EG after PDA coating. Remarkably, a maximum areal and volumetric capacitance as high as 2.04 $\mathrm{mF} \mathrm{cm}{ }^{-2}$ and $340 \mathrm{~F} \mathrm{~cm}^{-3}$ are delivered at $10 \mathrm{mV} \mathrm{s}^{-1}$ based on $60 \mathrm{~nm}$ thick PDA-EG electrode, which compares favorably to state-of-the-art MSCs/ supercapacitor performances based on graphene or other carbon materials, e.g. $212 \mathrm{~F} \mathrm{~cm}^{-3}$ based on holey graphene framework, ${ }^{39} 180 \mathrm{~F}$ $\mathrm{cm}^{-3}$ and $410 \mathrm{~F} \mathrm{~cm}^{-3}$ based on carbide-derived carbon, ${ }^{40-41} 1.3 \mathrm{~F} \mathrm{~cm}^{-3}$ based on onion-like carbon, ${ }^{42}$ $\sim 3 \mathrm{~F} \mathrm{~cm}^{-3}$ based on laser reduced $\mathrm{GO},,^{10-11} 376 \mathrm{~F} \mathrm{~cm}^{-3}$ based on high temperature annealing (1000 
${ }^{\circ} \mathrm{C}$ ) of graphene hydrogel, ${ }^{43} 359 \mathrm{~F} \mathrm{~cm}^{-3}$ based on selective electrophoretic deposited RGO, $261 \mathrm{~F}$ $\mathrm{cm}^{-3}$ based on liquid-mediated densely packed $\mathrm{RGO},{ }^{44} \sim 500 \mathrm{~F} \mathrm{~cm}^{-3}$ based on nitrogen-doped mesoporous carbon, ${ }^{45} 135 \mathrm{~F} \mathrm{~cm}^{-3}$ based on fluorine modified graphene, ${ }^{46}$ and $17.9 \mathrm{~F} \mathrm{~cm}^{-3}$ based on methane plasma treated GO..$^{38}$

The evolution of volumetric capacitance of PDA-EG-MSC-60 $\mathrm{nm}$ with consecutive scan rates is plotted in Figure 5a (CV curves of PDA-EG-MSC-120 nm shown in Fig. S9). Interestingly, the PDA-EG-MSC-60 nm exhibits an excellent rate capability with capacitance retention of 30\%, when the scan rate increases four orders of magnitude from 10 to $10^{5} \mathrm{mV} \mathrm{s}^{-1}$. Such rate capability guarantees a promising power density of the MSC under high operating speeds. As expected, the volumetric capacitance decreases with increasing film thickness, which can be partially attributed to the limited interlayer conductivity of the PDA-EG films. Moreover, in Figure 5b, the logarithmic discharge current exhibits a nearly linear dependence upon scan rates from 10 to $10^{5} \mathrm{mV} \mathrm{s}^{-1}$, suggesting the ultrahigh power delivery of PDA-EG-MSC. The Ragone plot in Figure 5c lists the volumetric energy/power densities of our PDA-EG-MSC together with those of commercially available high-energy lithium thin-film battery (4 V/500 $\mu \mathrm{Ah})$, and high-power aluminum electrolytic capacitor ( $3 \mathrm{~V} / 300 \mu \mathrm{F})$. The PDA-EG-MSC-60 $\mathrm{nm}$ provides energy densities of 2.78-9.44 $\mathrm{mWh} \mathrm{cm}^{-3}$, which are competitive with that of lithium thin-film battery (1$10 \mathrm{mWh} \mathrm{cm}^{-3}$ ). Furthermore, a maximum power density of $1000 \mathrm{~W} \mathrm{~cm}^{-3}$ is also delivered by the same device, which is two orders of magnitude higher than that of conventional sandwichedstructured supercapacitors and one order of magnitude higher than that of an aluminum electrolytic capacitor. ${ }^{13}$ Such direct comparisons demonstrate the great potential of PDA-EG based reliable and powerful ESSs. In addition, Figure 5d shows the cycling stability of PDA-EGMSC-60 nm, revealing a capacitance loss identified after 10000 cycles as low as 5\%. 


\section{Conclusion}

In summary, we demonstrated an efficient green chemistry approach to produce water-dispersed high-quality graphene nanosheets, via electrochemical exfoliation of graphite. The optimized exfoliation process guarantees the presence of sufficient oxygen-containing groups on the graphene surface, resulting in stable water dispersions, and thus opening up the possibilities for further functionalization in aqueous condition. Meanwhile, the electronic properties of graphene are maintained, as evidenced by XPS, Raman, conductivity, and Terahertz studies, and are remarkably better than RGO and highly comparable to the reported electrochemically exfoliated graphene via fast exfoliation. Our EG is likely to replace the dominant position of GO/RGO regarded as the water-dispersible form of graphene, highlighting the potential impact of EG, which to date has been limited by the lack dispersibility and thus processability. MSCs based on graphene flakes functionalized by homogenous PDA coating delivered a remarkable volumetric capacitance of $340 \mathrm{~F} \mathrm{~cm}^{-3}$ and excellent power density of $1000 \mathrm{~W} \mathrm{~cm}^{-3}$, which outperformed most of the reported MSCs based on graphene and highly comparable to the dominant commercially available high energy/power devices. Therefore, our EG combines all the desired characteristics which have been pursued during the last decade in RGO. Further optimizations of the electrochemical exfoliation process may improve both the electronic properties and processabilities of this novel EG, and by using concepts well-established for the in situ functionalization of RGO, various multifunctional composite materials such as conducting polymers-EG, nanoparticles-EG, for energy conversion and storage, optoelectronics, bio-sensing, etc, may be developed. 
Figures

(a)
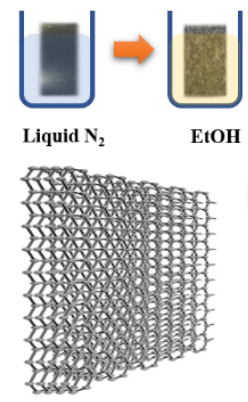

Pre-treated Graphite

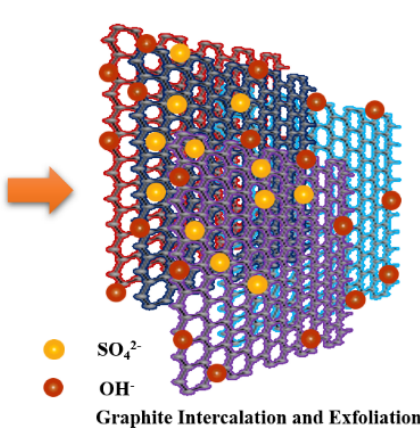

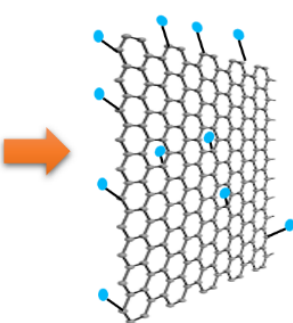

Water-dispersed Graphen

(d)

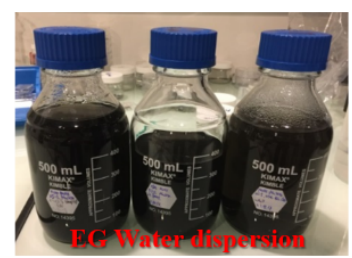

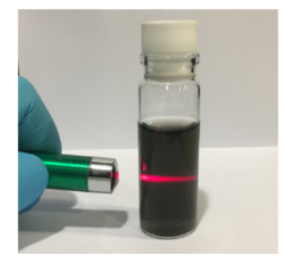

(e)

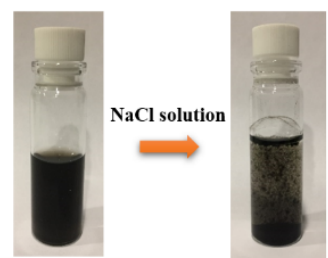
mixed electrolyte

Figure 1. Electrochemical exfoliation of graphite and the obtained graphene dispersions in water. a) Schematic illustration of the electrochemical exfoliation process employed towards water-dispersed graphene and subsequent functionalization with polydopamine. b) Experimental setup for electrochemical exfoliation of graphite. c) Photographs of graphene dispersion in water. d) Tyndall effect of graphene water dispersion. e) Salt effect of graphene water dispersion, which suggests by the addition of sodium chloride, the stabilization of the graphene water dispersion is severely disrupted. 


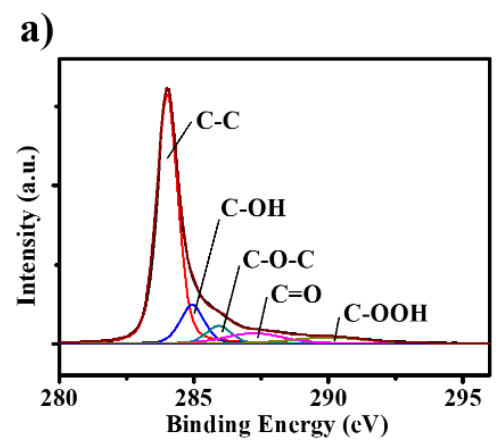

d)
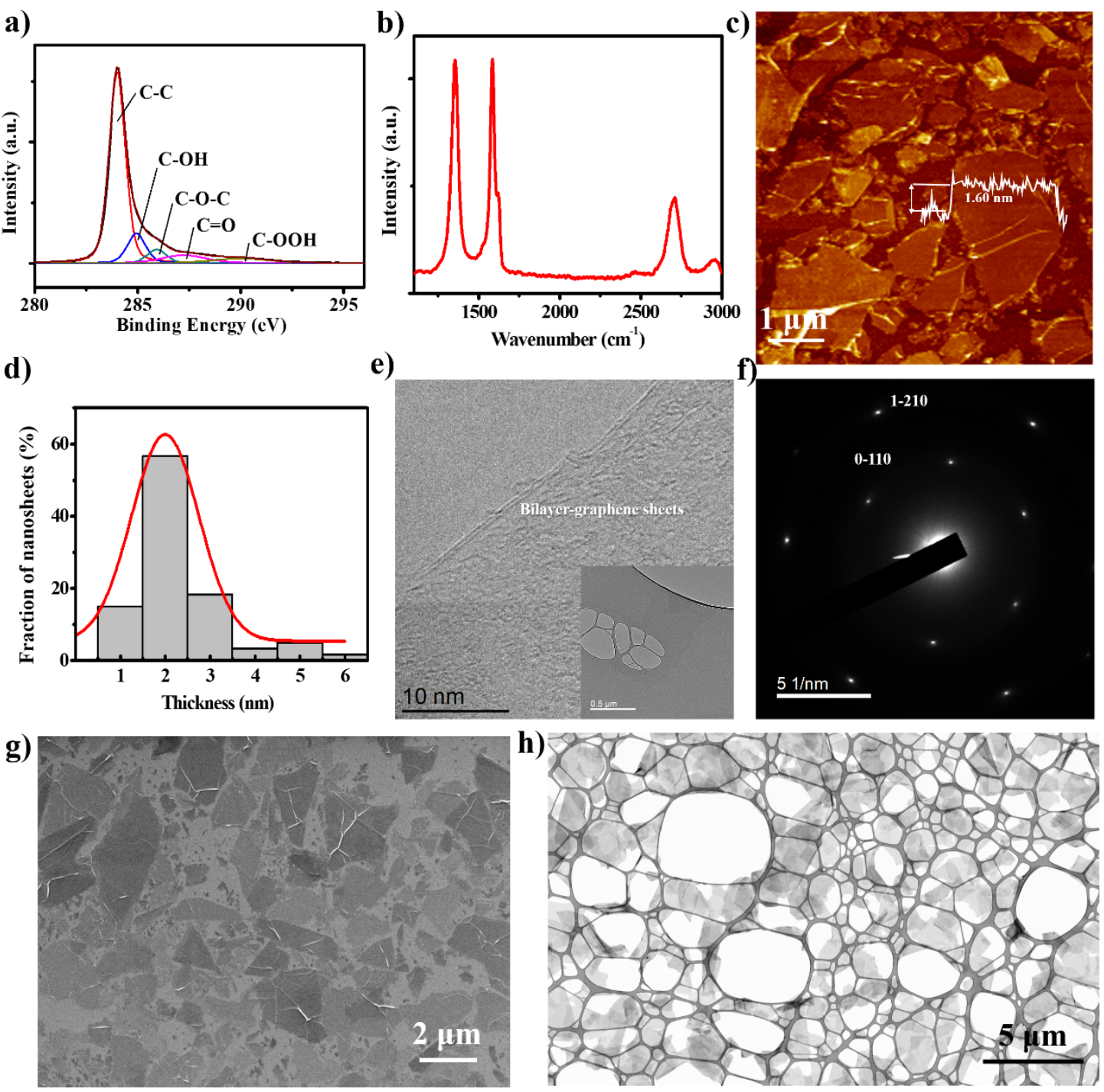

Figure 2. Spectroscopy and morphological characterizations of obtained graphene flakes. a) High-resolution XPS of the C 1s spectrum of EG. b) Raman spectra (excited by $532 \mathrm{~nm}$ laser) of a bilayer-flake of EG. c) AFM image of EG on $\mathrm{SiO} 2$ substrates. d) Statistical study of the thickness distribution of EG by AFM. e) HR-TEM images of EG, inset shows the selected flake with a large field of view. f) SAED pattern of the corresponding EG fragment. g) SEM image h) Typical TEM image of the specimen deposited on a TEM grid, with a large field of view. 
(a)

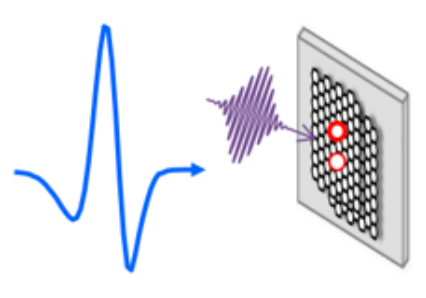

(c) 3
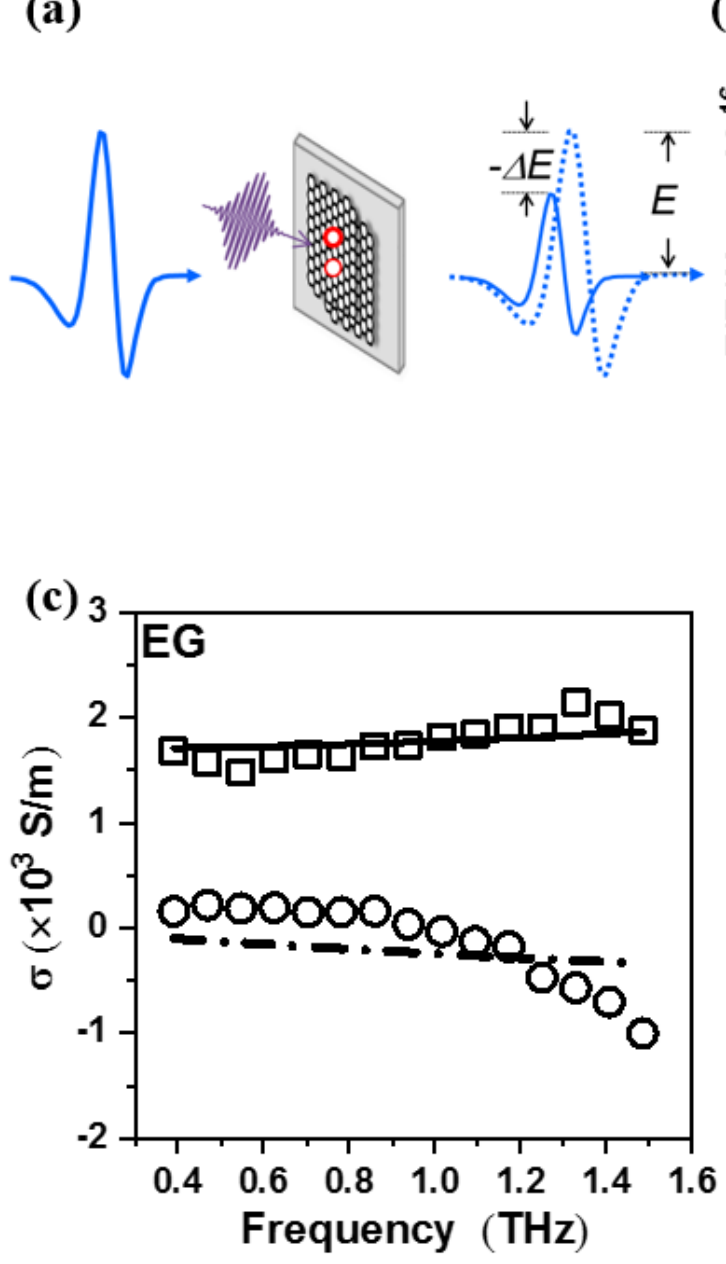

(b)

(d)

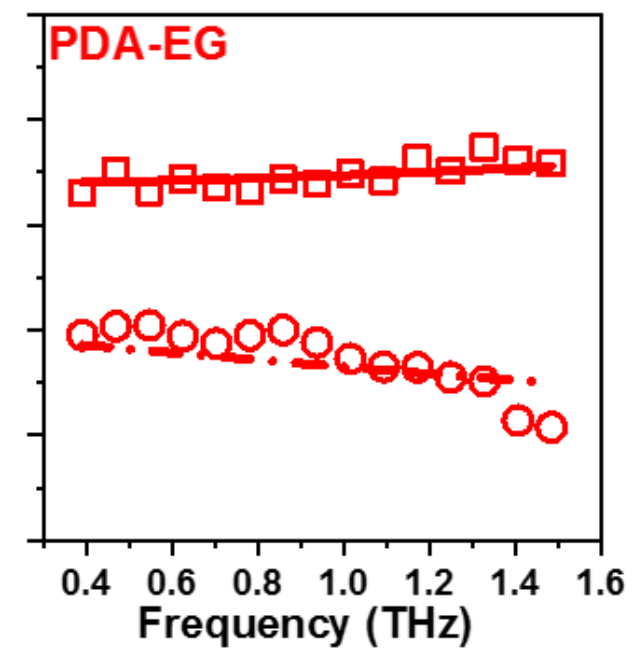

Figure 3. THz studies on the ultrafast charge carrier dynamics in graphene and polydopaminegraphene. a) illustrates the optical-pump $\mathrm{THz}$ probe spectroscopy. b) compares the ultrafast $\mathrm{THz}$ conductivity in both samples. The $\mathrm{THz}$ conductivity is normalized to the absorbed photon densities to allow for a quantitative comparison. The inset shows the fluence dependent $\mathrm{THz}$ conductivities. c-d) show the frequency conductivity of photogenerated carriers and corresponding Drude-Smith model calculations, from which the charge carrier scattering time, and corresponding mobility is inferred. 

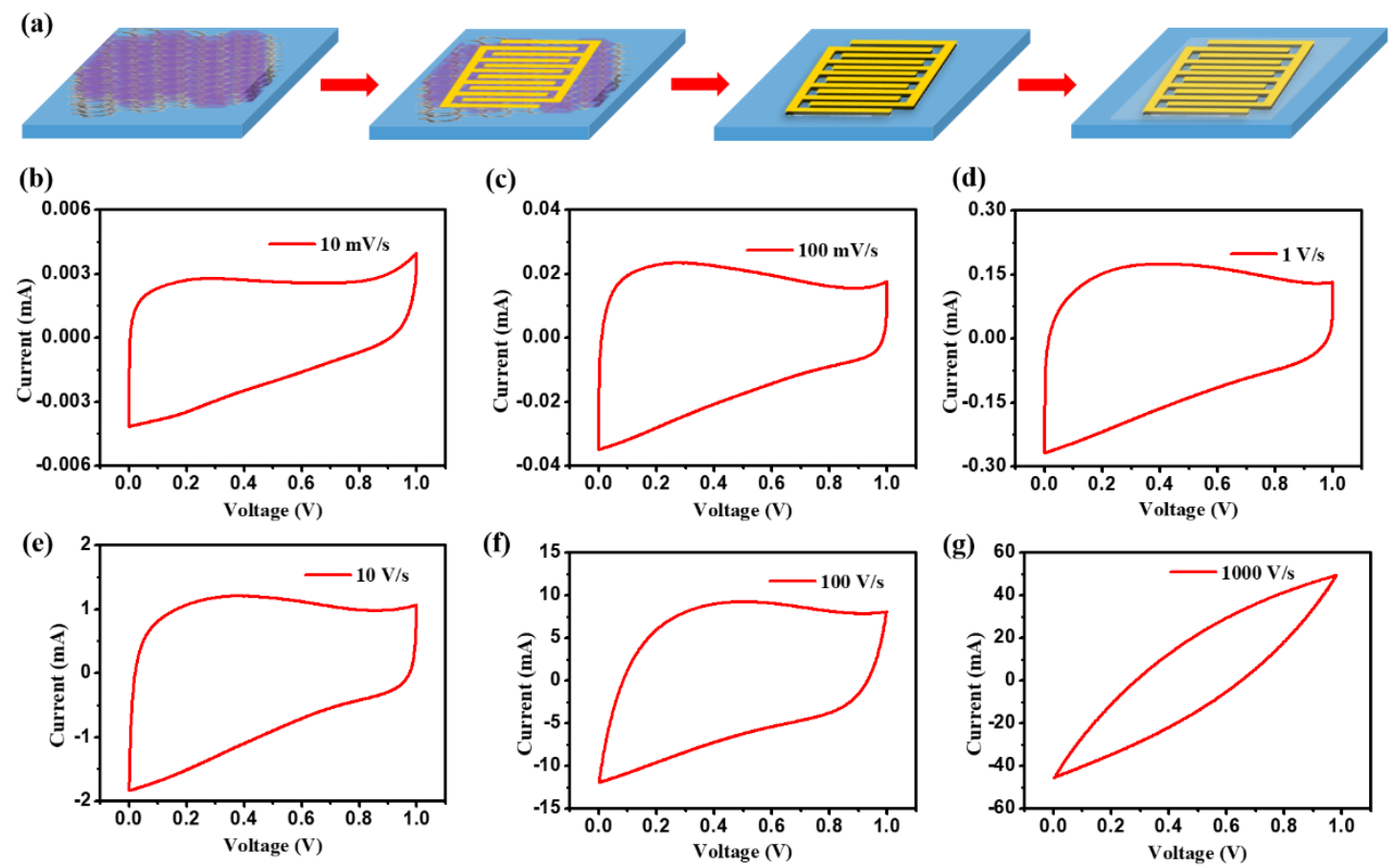

Figure 4. Polydopamine-graphene based micro-supercapacitor device fabrication and characterization. a) Schematic illustration of the device fabrication of MSCs based on PDA-EG electrodes on silicon wafer. b-g) CV curves of PDA-EG-MSC-60 nm performed at different scan rates ranging from $10 \mathrm{mV} \mathrm{s}-1$ to $1000 \mathrm{~V} \mathrm{~s}-1$, with potential window from 0 to $1 \mathrm{~V}$. 
(a)

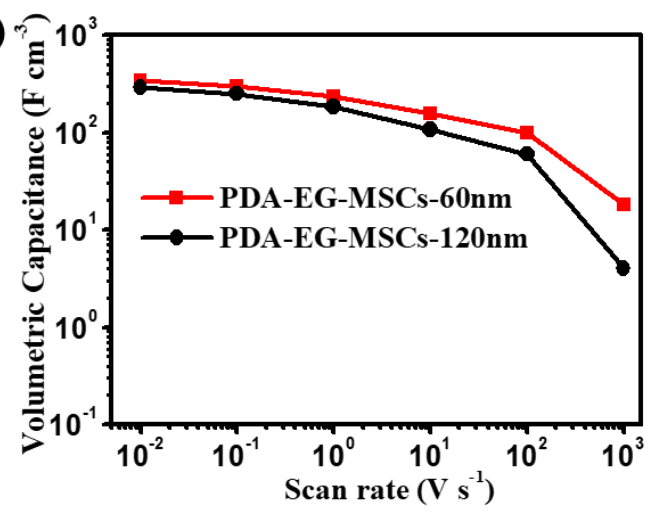

(c)

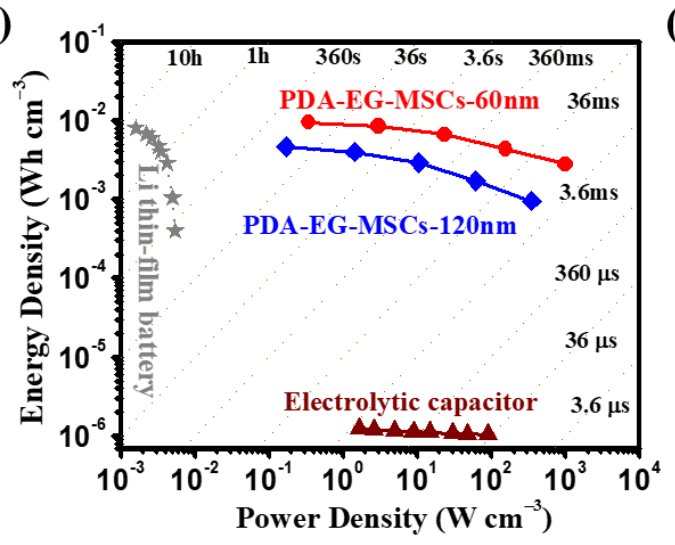

(b)

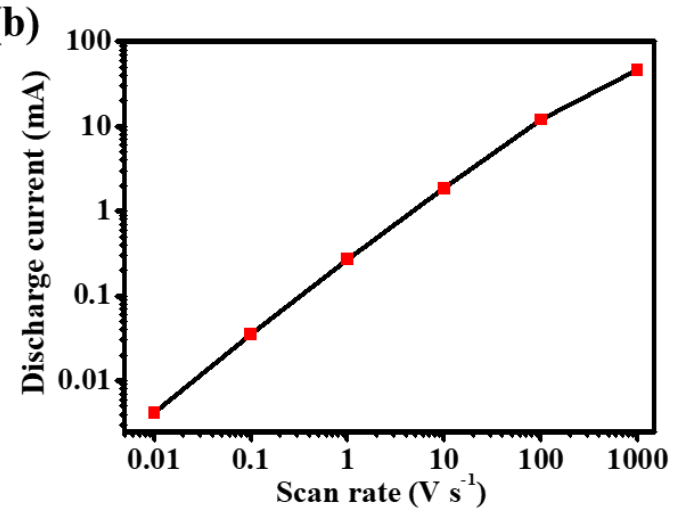

(d)

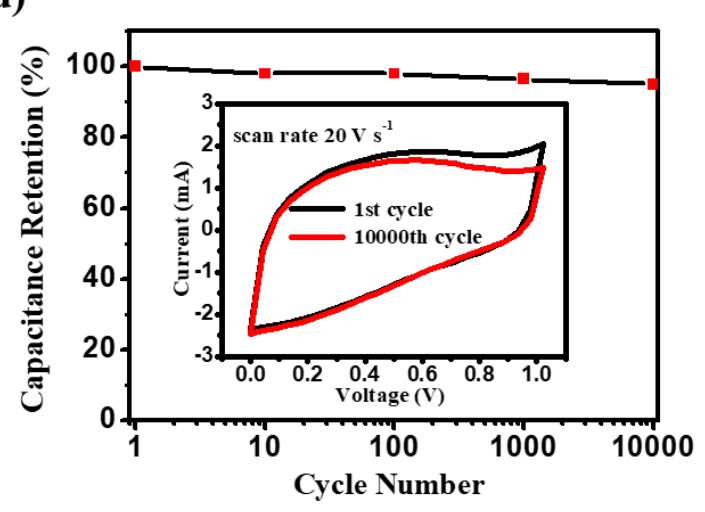

Figure 5. Summary of electrochemical performance of polydopamine-graphene microsupercapacitors. a) Evolution of the volumetric capacitance versus scan rate based on two different PDA-EG film thicknesses. b) Discharge current as a function of scan rate based on PDA-EG-MSC-60 nm device. c) Ragone plot of commercial Li thin-film battery, Electrolytic capacitor, and PDA-EG-MSC based on two different PDA-EG film thickness. d) cycling stability of PDA-EG-MSC-60 nm device over 10000 cycles, at scan rate $20 \mathrm{~V} \mathrm{~s}^{-1}$. Inset shows the CV curves of the $1^{\text {st }}$ and $10000^{\text {th }}$ cycle. 


\section{ASSOCIATED CONTENT}

Supporting Information. Detailed Experimental sections and supporting figures are available in the Supporting Information.

\section{AUTHOR INFORMATION}

\section{Corresponding Author}

Artur Ciesielski ciesielski@unistra.fr; Paolo Samorì samori@ unistra.fr

\section{Author Contributions}

Z.L., A.C., and P.S. conceived and designed the project. Z.L. performed the electrochemical exfoliation of graphite, and the subsequent processing and characterization of obtained graphene nanosheets, as well as the micro-supercapacitor fabrication and evaluation. H.Z., M.B., and H.I.W. performed THz measurements. M.E. performed the TEM, SEM and XPS study. W.B. and O.E. performed the high-resolution TEM measurements. H.Q. involved in the electrical characterization of EG.

\section{Notes}

The authors declare no competing financial interest.

\section{ACKNOWLEDGMENT}

Dr. Rafael Furlan de Oliveira and Dr. Yifan Yao are acknowledged for enlightening discussions.

Dr. Simone Silvestrini is acknowledged for the help of Z-potential measurements. We acknowledge the financial support by the European Commission through the Graphene Flagship Core 2 project (GA No. 785219), the AMI project funded by the ERA-NET EuroNanoMed III 
programme, the European Union and the Agence Nationale de la Recherche (ANR) GA-ANR17-ENM3-0001-01, the ANR through the Labex project CSC (ANR-10-LABX-0026 CSC) within the Investissement d'Avenir program (ANR-10-120 IDEX-0002-02), and the International Center for Frontier Research in Chemistry (icFRC).

\section{REFERENCES}

1. Bonaccorso, F.; Colombo, L.; Yu, G.; Stoller, M.; Tozzini, V.; Ferrari, A. C.; Ruoff, R. S.; Pellegrini, V., Graphene, related two-dimensional crystals, and hybrid systems for energy conversion and storage. Science 2015, 347, 1246501 .

2. Raccichini, R.; Varzi, A.; Passerini, S.; Scrosati, B., The role of graphene for electrochemical energy storage. Nat. Mater. 2015, 14, 271-279.

3. Kyeremateng, N. A.; Brousse, T.; Pech, D., Microsupercapacitors as miniaturized energystorage components for on-chip electronics. Nat. Nano. 2017, 12, 7-15.

4. Wu, Z.-S.; Wang, D.-W.; Ren, W.; Zhao, J.; Zhou, G.; Li, F.; Cheng, H.-M., Anchoring Hydrous RuO2 on Graphene Sheets for High-Performance Electrochemical Capacitors. Adv. Funct. Mater. 2010, 20, 3595-3602.

5. Yu, G.; Xie, X.; Pan, L.; Bao, Z.; Cui, Y., Hybrid nanostructured materials for highperformance electrochemical capacitors. Nano Energy 2013, 2, 213-234.

6. $\quad$ Liu, S.; Gordiichuk, P.; Wu, Z.-S.; Liu, Z.; Wei, W.; Wagner, M.; Mohamed-Noriega, N.; Wu, D.; Mai, Y.; Herrmann, A.; Mullen, K.; Feng, X., Patterning two-dimensional free-standing surfaces with mesoporous conducting polymers. Nat. Commun. 2015, 6, 8817. 
7. Meng, Y.; Wang, K.; Zhang, Y.; Wei, Z., Hierarchical Porous Graphene/Polyaniline Composite Film with Superior Rate Performance for Flexible Supercapacitors. Adv. Mater. 2013, 25, 6985-6990.

8. Wang, D.-W.; Li, F.; Zhao, J.; Ren, W.; Chen, Z.-G.; Tan, J.; Wu, Z.-S.; Gentle, I.; Lu, G. Q.; Cheng, H.-M., Fabrication of Graphene/Polyaniline Composite Paper via In Situ Anodic Electropolymerization for High-Performance Flexible Electrode. ACS nano 2009, 3, 1745-1752.

9. $\quad$ El-Kady, M. F.; Strong, V.; Dubin, S.; Kaner, R. B., Laser Scribing of High-Performance and Flexible Graphene-Based Electrochemical Capacitors. Science 2012, 335, 1326-1330.

10. El-Kady, M. F.; Kaner, R. B., Scalable fabrication of high-power graphene microsupercapacitors for flexible and on-chip energy storage. Nat. Commun. 2013, 4, 1475.

11. Gao, W.; Singh, N.; Song, L.; Liu, Z.; Reddy, A. L. M.; Ci, L.; Vajtai, R.; Zhang, Q.; Wei, B.; Ajayan, P. M., Direct laser writing of micro-supercapacitors on hydrated graphite oxide films. Nat. Nano. 2011, 6, 496-500.

12. Niu, Z.; Zhang, L.; Liu, L.; Zhu, B.; Dong, H.; Chen, X., All-Solid-State Flexible Ultrathin Micro-Supercapacitors Based on Graphene. Adv. Mater. 2013, 25, 4035-4042.

13. Wu, Z. S.; Parvez, K.; Feng, X.; Müllen, K., Graphene-based in-plane microsupercapacitors with high power and energy densities. Nat. Commun. 2013, 4, 2487.

14. Yang, S.; Lohe, M. R.; Müllen, K.; Feng, X., New-Generation Graphene from Electrochemical Approaches: Production and Applications. Adv. Mater. 2016, 28, 6213-6221. 
15. Parvez, K.; Wu, Z.-S.; Li, R.; Liu, X.; Graf, R.; Feng, X.; Müllen, K., Exfoliation of Graphite into Graphene in Aqueous Solutions of Inorganic Salts. J. Am. Chem. Soc. 2014, 136, 6083.

16. Yang, S.; Brüller, S.; Wu, Z.-S.; Liu, Z.; Parvez, K.; Dong, R.; Richard, F.; Samorì, P.; Feng, X.; Müllen, K., Organic Radical-Assisted Electrochemical Exfoliation for the Scalable Production of High-Quality Graphene. J. Am. Chem. Soc. 2015, 137, 13927-13932.

17. Wang, Y.; Liu, Y.; Zhang, J.; Wu, J.; Xu, H.; Wen, X.; Zhang, X.; Tiwary, C. S.; Yang, W.; Vajtai, R.; Zhang, Y.; Chopra, N.; Odeh, I. N.; Wu, Y.; Ajayan, P. M., Cryo-mediated exfoliation and fracturing of layered materials into 2D quantum dots. Sci. Adv. 2017, 3, 1701500.

18. Parvez, K.; Li, R.; Puniredd, S. R.; Hernandez, Y.; Hinkel, F.; Wang, S.; Feng, X.; Müllen, K., Electrochemically Exfoliated Graphene as Solution-Processable, Highly Conductive Electrodes for Organic Electronics. ACS nano 2013, 7, 3598-3606.

19. Parvez, K.; Wu, Z.-S.; Li, R.; Liu, X.; Graf, R.; Feng, X.; Müllen, K., Exfoliation of Graphite into Graphene in Aqueous Solutions of Inorganic Salts. J. Am. Chem. Soc. 2014, 136, 6083-6091.

20. Li, D.; Muller, M. B.; Gilje, S.; Kaner, R. B.; Wallace, G. G., Processable aqueous dispersions of graphene nanosheets. Nat. Nano. 2008, 3, 101-105.

21. Voiry, D.; Yang, J.; Kupferberg, J.; Fullon, R.; Lee, C.; Jeong, H. Y.; Shin, H. S.; Chhowalla, M., High-quality graphene via microwave reduction of solution-exfoliated graphene oxide. Science 2016, 353, 1413-1416. 
22. Bagri, A.; Mattevi, C.; Acik, M.; Chabal, Y. J.; Chhowalla, M.; Shenoy, V. B., Structural evolution during the reduction of chemically derived graphene oxide. Nat. Chem. 2010, 2, 581587.

23. Su, C. Y.; Lu, A. Y.; Xu, Y. P.; Chen, F. R.; Khlobystov, A. N.; Li, L. J., High-Quality Thin Graphene Films from Fast Electrochemical Exfoliation. ACS nano 2011, 5, 2332-2339.

24. Miao, F.; Majee, S.; Song, M.; Zhao, J.; Zhang, S.-L.; Zhang, Z.-B., Inkjet printing of electrochemically-exfoliated graphene nano-platelets. Synth. Met. 2016, 220, 318-322.

25. Eredia, M.; Bertolazzi, S.; Leydecker, T.; El Garah, M.; Janica, I.; Melinte, G.; Ersen, O.; Ciesielski, A.; Samorì, P., Morphology and Electronic Properties of Electrochemically Exfoliated Graphene. J. Phys. Chem. Lett. 2017, 8, 3347-3355.

26. Buglione, L.; Chng, E. L. K.; Ambrosi, A.; Sofer, Z.; Pumera, M., Graphene materials preparation methods have dramatic influence upon their capacitance. Electrochem. Commun. $2012,14,5-8$.

27. Cheng, Z.; Zhou, Q.; Wang, C.; Li, Q.; Wang, C.; Fang, Y., Toward Intrinsic Graphene Surfaces: A Systematic Study on Thermal Annealing and Wet-Chemical Treatment of SiO2Supported Graphene Devices. Nano Lett. 2011, 11, 767-771.

28. Hu, L.; Choi, J. W.; Yang, Y.; Jeong, S.; La Mantia, F.; Cui, L.-F.; Cui, Y., Highly conductive paper for energy-storage devices. PNAS USA 2009, 106, 21490-21494. 
29. Wang, H. I.; Braatz, M.-L.; Richter, N.; Tielrooij, K.-J.; Mics, Z.; Lu, H.; Weber, N.-E.; Müllen, K.; Turchinovich, D.; Kläui, M.; Bonn, M., Reversible Photochemical Control of Doping Levels in Supported Graphene. J. Phys. Chem. C 2017, 121, 4083-4091.

30. Ulbricht, R.; Hendry, E.; Shan, J.; Heinz, T. F.; Bonn, M., Carrier dynamics in semiconductors studied with time-resolved terahertz spectroscopy. Rev. Mod. Phys 2011, 83, 543-586.

31. Tomadin, A.; Hornett, S. M.; Wang, H. I.; Alexeev, E. M.; Candini, A.; Coletti, C.; Turchinovich, D.; Kläui, M.; Bonn, M.; Koppens, F. H. L.; Hendry, E.; Polini, M.; Tielrooij, K.J., The ultrafast dynamics and conductivity of photoexcited graphene at different Fermi energies. Sci. Adv. 2018, 4, 5313.

32. Wang, H. I.; Infante, I.; Brinck, S.t.; Cánovas, E.; Bonn, M., Efficient Hot Electron Transfer in Quantum Dot-Sensitized Mesoporous Oxides at Room Temperature. Nano Lett. $2018,18,5111-5115$.

33. Chen, Z.; Wang, H. I.; Bilbao, N.; Teyssandier, J.; Prechtl, T.; Cavani, N.; Tries, A.; Biagi, R.; De Renzi, V.; Feng, X.; Kläui, M.; De Feyter, S.; Bonn, M.; Narita, A.; Müllen, K., Lateral Fusion of Chemical Vapor Deposited N = 5 Armchair Graphene Nanoribbons. J. Am. Chem. Soc. 2017, 139, 9483-9486.

34. Tielrooij, K. J.; Song, J. C. W.; Jensen, S. A.; Centeno, A.; Pesquera, A.; Zurutuza Elorza, A.; Bonn, M.; Levitov, L. S.; Koppens, F. H. L., Photoexcitation cascade and multiple hot-carrier generation in graphene. Nat. Phys. 2013, 9, 248-252. 
35. Su, Y.; Yao, Z.; Zhang, F.; Wang, H.; Mics, Z.; Cánovas, E.; Bonn, M.; Zhuang, X.; Feng, X., Sulfur-Enriched Conjugated Polymer Nanosheet Derived Sulfur and Nitrogen coDoped Porous Carbon Nanosheets as Electrocatalysts for Oxygen Reduction Reaction and ZincAir Battery. Adv. Funct. Mater. 2016, 26, 5893-5902.

36. Nurlaela, E.; Wang, H.; Shinagawa, T.; Flanagan, S.; Ould-Chikh, S.; Qureshi, M.; Mics, Z.; Sautet, P.; Le Bahers, T.; Cánovas, E.; Bonn, M.; Takanabe, K., Enhanced Kinetics of Hole Transfer and Electrocatalysis during Photocatalytic Oxygen Evolution by Cocatalyst Tuning. ACS Catal. 2016, 6, 4117-4126.

37. Ferrari, A. C.; Bonaccorso, F.; Fal'ko, V.; Novoselov, K. S.; Roche, S.; Boggild, P.; Borini, S.; Koppens, F. H. L.; Palermo, V.; Pugno, N.; Garrido, J. A.; Sordan, R.; Bianco, A.; Ballerini, L.; Prato, M.; Lidorikis, E.; Kivioja, J.; Marinelli, C.; Ryhanen, T.; Morpurgo, A.; Coleman, J. N.; Nicolosi, V.; Colombo, L.; Fert, A.; Garcia-Hernandez, M.; Bachtold, A.; Schneider, G. F.; Guinea, F.; Dekker, C.; Barbone, M.; Sun, Z.; Galiotis, C.; Grigorenko, A. N.; Konstantatos, G.; Kis, A.; Katsnelson, M.; Vandersypen, L.; Loiseau, A.; Morandi, V.; Neumaier, D.; Treossi, E.; Pellegrini, V.; Polini, M.; Tredicucci, A.; Williams, G. M.; Hee Hong, B.; Ahn, J.-H.; Min Kim, J.; Zirath, H.; van Wees, B. J.; van der Zant, H.; Occhipinti, L.; Di Matteo, A.; Kinloch, I. A.; Seyller, T.; Quesnel, E.; Feng, X.; Teo, K.; Rupesinghe, N.; Hakonen, P.; Neil, S. R. T.; Tannock, Q.; Lofwander, T.; Kinaret, J., Science and technology roadmap for graphene, related two-dimensional crystals, and hybrid systems. Nanoscale 2015, 7, 4598-4810.

38. Wu, Z. S.; Parvez, K.; Feng, X.; Müllen, K., Graphene-based in-plane microsupercapacitors with high power and energy densities. Nat. Commun. 2013, 4, 2487. 
39. Xu, Y.; Lin, Z.; Zhong, X.; Huang, X.; Weiss, N. O.; Huang, Y.; Duan, X., Holey graphene frameworks for highly efficient capacitive energy storage. Nat. Commun. 2014, 5, 4554.

40. Chmiola, J.; Largeot, C.; Taberna, P.-L.; Simon, P.; Gogotsi, Y., Monolithic CarbideDerived Carbon Films for Micro-Supercapacitors. Science 2010, 328, 480-483.

41. Huang, P.; Lethien, C.; Pinaud, S.; Brousse, K.; Laloo, R.; Turq, V.; Respaud, M.; Demortière, A.; Daffos, B.; Taberna, P. L.; Chaudret, B.; Gogotsi, Y.; Simon, P., On-chip and freestanding elastic carbon films for micro-supercapacitors. Science 2016, 351, 691-695.

42. Pech, D.; Brunet, M.; Durou, H.; Huang, P.; Mochalin, V.; Gogotsi, Y.; Taberna, P.-L.; Simon, P., Ultrahigh-power micrometre-sized supercapacitors based on onion-like carbon. Nat. Nano. 2010, 5, 651-654.

43. Tao, Y.; Xie, X.; Lv, W.; Tang, D.-M.; Kong, D.; Huang, Z.; Nishihara, H.; Ishii, T.; Li, B.; Golberg, D.; Kang, F.; Kyotani, T.; Yang, Q.-H., Towards ultrahigh volumetric capacitance: graphene derived highly dense but porous carbons for supercapacitors. Sci. Rep. 2013, 3, 2975.

44. Yang, X.; Cheng, C.; Wang, Y.; Qiu, L.; Li, D., Liquid-Mediated Dense Integration of Graphene Materials for Compact Capacitive Energy Storage. Science 2013, 341, 534-537.

45. Lin, T.; Chen, I.-W.; Liu, F.; Yang, C.; Bi, H.; Xu, F.; Huang, F., Nitrogen-doped mesoporous carbon of extraordinary capacitance for electrochemical energy storage. Science 2015, 350, 1508-1513. 
46. Zhou, F.; Huang, H.; Xiao, C.; Zheng, S.; Shi, X.; Qin, J.; Fu, Q.; Bao, X.; Feng, X.; Müllen, K.; Wu, Z.-S., Electrochemically Scalable Production of Fluorine-Modified Graphene for Flexible and High-Energy Ionogel-Based Microsupercapacitors. J. Am. Chem. Soc. 2018, $140,8198-8205$. 